\title{
Utilização de procedimentos de ensino-aprendizagem: Relatos de analistas do comportamento
}

\section{Using teaching-learning procedures: Behavior analysts reports}

Utilización de procedimientos de enseñanza-aprendizaje: Relatos de analistas de conducta

\author{
Marcos Spector Azoubel ${ }^{1}$, Mônica Helena Tieppo Alves Gianfaldoni²
}

[1] [2] Pontifícia Universidade Católica de São Paulo I Título abreviado: I Endereço para correspondência:Endereço para correspondência: Laboratório de Psicologia Experimental: Análise do Comportamento, Rua Bartira, 387, CEP 05009-000 - São Paulo - SP | Email: (Marcos) mazoubel@gmail.com I (Mônica) mhtag@pucsp.com.br

Resumo: Há diversos estudos de analistas do comportamento sobre educação. Deles, derivaram-se diversos procedimentos de ensino. Apesar de terem eficácia demonstrada por meio de pesquisas empíricas, são pouco utilizados. Apontam-se diversas causas para tal desuso e a maior parte delas diz respeito a pré-conceitos, ignorância ou não aceitação de propostas de autores da área. Para investigar possíveis variáveis envolvidas na utilização de procedimentos de ensino derivados da Análise do Comportamento foram entrevistados cinco analistas do comportamento com experiência em orientação de estudos sobre educação. Os entrevistados apontaram como principais barreiras para uso dos procedimentos da área: aumento do custo de resposta para o professor e rigidez de exigências das instituições de ensino. A maior parte dos obstáculos apontados para utilização dos procedimentos de ensino da área diz respeito a problemas inerentes às tecnologias e à própria comunidade. É questionada a afirmação de que concepções errôneas e ignorância sobre Análise do Comportamento são as principais responsáveis para o desuso dos procedimentos considerados comportamentais para o ensino. Todos os participantes relataram dificuldades em utilizar procedimentos da área; assim, não são utilizados os procedimentos de forma integral, mas adaptações dos mesmos. Apesar de haver consenso quanto à eficácia de tais adaptações, ele não tem sido suficiente para manutenção das mesmas.

Palavras-chave: práticas baseadas em evidências, procedimentos de ensino, tecnologia de ensino 
Abstract: There are many behavior analysts' studies about education. Several teaching procedures are derived from them. Despite demonstrated effectiveness by empirical research, are underutilized. Several causes for such disuse are pointed out, most of them with respect to preconceptions, ignorance or rejection of the authors of the field proposals. To investigate possible variables involved in the use of teaching procedures derived from the Behavior Analysis, five behavior analysts, with experience in the supervision of educational researches, were interviewed. They pointed out as the main obstacles: increased response cost to the teacher and stiffness requirements of educational institutions. Most obstacles mentioned regards to problems inherent to the technology and the community. Therefore, it is placed into question the arguments pointing out misconceptions, and ignorance as major cause for the underutilization of behavior analysis' teaching procedures. All participants reported difficulties in using the teaching methods; therefore, they are not wholly utilized, but adaptations of them. Despite the consensus on the effectiveness of such adaptations, this has not been sufficient to maintain them.

Keywords: evidence-based practices, teaching procedures, teaching technology.

Resumen: Hay diversos estudios de analistas de la conducta sobre educación. Varios procedimientos de enseñanza se derivan de ellos. A pesar de la eficacia demostrada por la investigación empírica, son subutilizados. Varias causas de tal desuso se señalan, la mayoría de ellos con respecto a los prejuicios, la ignorancia o el rechazo de las propuestas de autores de la disciplina. Para investigar las posibles variables que intervienen en el uso de procedimientos de enseñanza derivadas del Análisis de Conducta, cinco analistas del comportamiento, con experiencia en supervisión de investigaciones educativas, fueron entrevistados. Señalaron como principales obstáculos: aumento del coste de respuesta del profesor y de rigidez de las instituciones educativas. La mayoría de los obstáculos mencionados se refiere a problemas inherentes a la tecnología y la comunidad. Por lo tanto, se cuestionan los argumentos que señalan los conceptos erróneos y la ignorancia como causa principal de la subutilización de los procedimientos de enseñanza del análisis de la conducta. Todos los participantes reportaron dificultades en el uso de los métodos de enseñanza; por lo tanto, no son totalmente utilizados, pero adaptaciones de las mismas. Incluso con el consenso sobre la efectividad de tales adaptaciones, esto no ha sido suficiente para mantenerlos.

Palabras-clave: prácticas basadas en evidencia, procedimientos de enseñanza, tecnología de la enseñanza. 
A preocupação com os caminhos da educação não é recente, nem circunscrita a uma área específica ou abordagem teórica em particular. Sua importância é evidente uma vez que planejar a educação é planejar o futuro da humanidade.

Para a Análise do Comportamento a educação é tema recorrente. Skinner (1989/1991) afirmou ter dedicado boa parte de sua obra para esse tema e, além dele, diversos outros analistas do comportamento dedicaram atenção ao assunto, tanto realizando pesquisas empíricas (Araújo, 2008; Crosbie \& Kelly, 1994; Miller \& Malott, 2006; Tudor, 1995) quanto propostas para utilização de conhecimentos sobre princípios básicos do comportamento nos procedimentos de ensino (Bijou, 1970; Cortegoso \& Coser, 2011; Holland, 1960; 1967; Juliano \& Luna, 2008; Keller, 1968; Keller \& Sherman, 1974; Kubo \& Botomé, 2001; Skinner, 1958; 1961; 1968; 1972; 1974a; 1974b; 1984).

$\mathrm{Na}$ perspectiva analítico-comportamental, ensinar é planejar e dispor contingências "para o desenvolvimento de comportamentos que serão vantajosos para o indivíduo e para outros em algum tempo futuro" (Skinner, 1953/2003, p. 437). Uma visão comprometida com essa ideia deve considerar a educação como englobando, ao mesmo tempo, o ensino por parte de professores e a aprendizagem por parte de alunos. Segundo Kubo e Botomé (2001), o ensino só pode ser analisado como uma relação entre comportamentos de quem ensina e de quem aprende, num processo ensinar-aprender. Desta forma, o ensino diz respeito às ações dos professores e aprender seria caracterizado pelas mudanças produzidas no aluno (aprendizado), decorrentes de tais ações dos professores. O ensino considerado eficaz seria aquele que, levando em conta os objetivos formulados, produzisse as alterações esperadas e importantes no repertório comportamental do aluno.

Interessados em eficácia do ensino, analistas do comportamento desenvolveram técnicas, métodos e procedimentos de ensino que formam a tecnologia analítico-comportamental do ensino. Fredrick e Hummel (2004) destacam Instrução Programada (IP), Precision Teaching (PT), Direct Instruction (DI) e o Sistema Personalizado de Ensino (PSI), como métodos com validade empírica comprovada. Na realidade brasileira, Nale (1998) ressalta a importância do método desenvolvido por Carolina Bori para programação de condições para o desenvolvimento de comportamentos (c.f., Kienen, Kubo \& Botomé, 2013). Pode-se acrescentar a essas tecnologias os estudos da área de equivalência de estímulos, que produziram um conjunto de procedimentos úteis para o ensino de vasta gama de comportamentos (Stromer, Mackay \& Stoddard, 1992), em especial para o ensino de comportamentos de ler e escrever, sendo tais métodos relevantes para o trabalho de analistas do comportamento em contextos educacionais.

A eficácia dos métodos analítico-comportamentais para a educação encontra fortes subsídios empíricos (Moran, 2004). Apesar disso, diversos autores indicam que não são utilizados em proporcional escala tais métodos no ensino regular (e. g. Deitz, 1994; Fantuzzo \& Atkins, 1992; Heward, 2005; Moreira, 2004; Rodrigues, 2005). Resta entender possíveis motivos dessa situação paradoxal.

Há, na literatura da área, diversas hipóteses sobre fatores que podem influenciar na dificuldade em difundir e aplicar os métodos de ensino produzidos pelos estudos de cientistas do comportamento (Tabela 1). Parte das possíveis explicações para a relativa insignificância dos efeitos de trabalhos analítico-comportamentais na educação regular costuma colocar isso na conta do desconhecimento ou discordâncias com as propostas da área (e.g., Rodrigues, 2005; 2012). Tais argumentos, porém, não são válidos para os próprios analistas do comportamento. Seria esperado que, ao menos, eles promovessem educação condizente com o que está estudado em relação a aprender e ensinar, mas isso parece não acontecer (Moreira, 2004).

Dada a pouca utilização de procedimentos de ensino da área (Moreira, 2004) o presente trabalho tem como objetivo investigar possíveis variáveis envolvidas na utilização de procedimentos de ensino derivados da Análise do Comportamento, em ensino regular na modalidade presencial, por parte de analistas do comportamento que orientaram teses e dissertações acerca das tecnologias comportamentais para educação. A escolha por investigar "práticas" de profissionais analistas do comportamento que são professores e têm experiência em orientação levou em conta a consideração de que devem conhecer profundamente as contribuições da área para a educação. 
Tabela 1. Aspectos Apresentados Como Possíveis Variáveis Que Dificultam na Difusão e na Aplicação de Métodos de Ensino da Área Encontrados na Literatura.

Artigo (s)
Skinner, 1958; 1984
Skinner, 1972; 1974a;
Rumph, Ninness,
McCuller, Holland, Ward,
\& Willbourn, 2007
Gioia, 2001; Miraldo,
1985; Rodrigues, 2005

Holland, 1978

Benjamin, 1988; Vargas \& Vargas, 1991; Skinner, 1963

Deitz, 1994 de em mudar a organização
Aspectos apresentados como possíveis variáveis

A sociedade não estaria preparada para conceber máquinas ensinando aos alunos e substituindo algumas atribuições do professor, haveria uma inércia cultural.

A concepção de educação baseada em princípios científicos entraria em conflito com visões tradicionais da pedagogia

Concepções distorcidas das contribuições da análise do comportamento e de propostas do behaviorismo radical são encontradas em diversos meios e parecem exercer influência na difusão dos métodos de ensino, técnicas e procedimentos da área.

Aqueles que trabalham com educação poderiam não ser capazes de colocar em prática métodos que alterem o establishment educacional. O psicólogo atende às demandas de seus clientes, chefes, governantes ou quem quer que o contrate, além de seguir objetivos traçados por eles. Assim, haveria dificuldaestabelecida de tal forma que o analista do comportamento pode ser encarado como parte do problema.

Aplicações de métodos analítico-comportamentais pouco eficazes também podem ter atrapalhado o processo de aceitação das aplicações da área.

A produção de dados sobre a eficácia das práticas educacionais derivadas da Análise do Comportamento não produzirá, necessariamente, a utilização destes métodos na educação. Afinal, os políticos e os mantenedores das instituições de ensino não costumam se importar com dados empíricos e quem os leva em consideração para decidir qual prática assumir são os cientistas.
Deitz, 1994

Analistas do comportamento por vezes extrapolariam os dados produzidos pelas pesquisas da área, afirmando terem dados suficientes para demonstrar que a Análise do Comportamento tem todas as soluções para a educação. Apesar de larga produção de conhecimento sobre diversas técnicas e princípios, existiriam muitos assuntos ainda inexplorados ou com poucas conclusões.

Benjamin, 1988; Keller, 1983; Teixeira, 2006

Haveria aumento a carga de trabalho do profissional que utiliza métodos de ensino da área em comparação com aqueles que não o fazem.

Axelrod, 1992; Fantuzzo \& Atkins, 1992

Problemas para implementação de procedimentos comportamentais poderiam surgir pelo fato de a maior parte dos procedimentos terem sido desenvolvidos em situações especiais de sala de aula, com tempo e quantidade de alunos reduzidos. As mesmas técnicas poderiam ter sua aplicação extremamente dificultada em condições naturais de ensino.

Miraldo, 1985; Axelrod, O linguajar técnico da área 1992; Fantuzzo \& Atkins, pode ser outra variável impor1992 tante para a dificuldade de entendimento e não aceitação das propostas comportamentais para a educação. Termos como controle, reforçamentos positivo e negativo ou punição podem facilitar a rejeição dos conceitos comportamentais.

\section{Método}

\section{Seleção de Informantes}

Segundo Luna (1998), é preciso ter certos cuidados especiais na escolha dos participantes de uma pesquisa quando o fenômeno for estudado por meio de relatos verbais. Tais participantes devem deter a informação, ser capazes de falar sobre elas e estar dispostos a fazê-lo ao pesquisador. Com objetivo de identificar nomes de analistas do comportamento que tenham pesquisado, além de terem orientado largamente estudos em educação e tecnologia do ensino e que trabalham ou tenham trabalhado com ensino regular, selecionaram-se os principais 
orientadores dos programas de pós-graduação com linhas de pesquisa em Análise do Comportamento.

Para encontrar esses orientadores de estudos envolvendo Análise do Comportamento e ensino, foi realizada uma busca com adaptação dos termos utilizados por Lacerda (2008) numa caracterização do ensino programado no Brasil. Os termos pesquisados foram: sistema personalizado de ensino, sistema programado individualizado, método Keller, curso individualizado, curso personalizado individualizado, curso programado individualizado, ensino individualizado, programa de ensino individualizado, instrução personalizada, personalized system of instruction, instrução programada, ensino programado individualizado, programação de ensino, sistema personalizado de instrução, planejamento de ensino, sistema de ensino, direct instruction, instrução direta, precision teaching, e ensino de precisão.

A presente pesquisa foi restrita às instituições de pós-graduação com linhas de pesquisa na área da Análise do Comportamento. Essas instituições foram previamente selecionadas na dissertação de mestrado de Santos (2012) e foram: PUCSP, UFSCar, UFPA, UNB, USP, UEL, Mackenzie, PUCCamp, UCB, PUC-GO, UFG, UFES, Unifesp, UFMG, UFPB, UFPR, UFSC, Unesp, Unicamp, e USP/RP.

O procedimento de busca foi realizado no Banco de Teses e Dissertações da CAPES (http:// capesdw.capes.gov.br/). Primeiramente, foi selecionada a opção "busca avançada" e, então, foram colocados os termos, um de cada vez, na primeira lacuna, com as opções "Todos os campos" e "é (exato)" marcadas. No segundo campo, foram digitados os nomes completos das instituições de ensino selecionadas, um a um, também com a seleção das opções “Todos os campos” e "é (exato)”. Para cada uma das instituições foram realizadas 20 buscas, uma para cada termo selecionado.

Esse primeiro passo resultou em 33 nomes de orientadores pertencentes a 11 instituições de ensino. $\mathrm{O}$ segundo passo foi o acesso aos currículos Lattes desses profissionais, a partir da busca textual na Plataforma Lattes do CNPQ (http://buscatextual.cnpq.br/buscatextual/busca. do?metodo=apresentar). Foram excluídos aqueles pesquisadores que não mencionaram: análise do comportamento, análise experimental do comportamento, psicologia comportamental, psicologia experimental ou análise experimental do comportamento nas suas apresentações. Ao utilizar este critério de exclusão, restaram 14 orientadores.

A última etapa consistiu na exclusão de profissionais com menos de 10 teses ou dissertações orientadas com os termos educação e ensino. É preciso esclarecer que tais termos não foram utilizados na busca inicial por serem amplos, mas seus usos são justificados quando todos os potenciais participantes são analistas do comportamento com experiência em orientação de temas sobre educação. Feito isso, restaram sete nomes de profissionais da área.

Finalmente, seis desses analistas do comportamento foram contatados para serem participantes da pesquisa. A opção por não convidar todos os informantes selecionados foi feita para dificultar a identificação de cada participante. Mesmo que os nomes dos sete potenciais participantes possam ser encontrados refazendo o procedimento de busca dos pesquisadores, não é possível saber quais, exatamente, foram convidados a participar da pesquisa.

\section{Participantes}

Dos seis pesquisadores convidados, foi possível realizar entrevistas com cinco analistas do comportamento que trabalham em quatro diferentes instituições públicas ou privadas de ensino como docentes de pós-graduação stricto senso.

\section{Procedimento}

As entrevistas foram conduzidas tanto presencialmente quanto por Skype, de acordo com a disponibilidade de cada participante. A pergunta inicial foi: "Na sua prática como professor, quais métodos de ensino derivados da análise do comportamento foram utilizados?". Um roteiro de perguntas foi previamente construído para evocar relatos sobre as categorias de análise do estudo, apresentados no próximo subtítulo; foi utilizado na medida em que os aspectos importantes para responder ao problema de pesquisa não tivessem sido abordados. Dessa forma, o roteiro funcionou como um guia para o pesquisador e possibilitou que informações adicionais pudessem ser solicitadas. 
Análise das entrevistas. As entrevistas foram gravadas e transcritas de forma literal. A partir das transcrições dos relatos dos participantes foram feitos exames iniciais em etapas. A primeira etapa consistiu uma leitura inicial com objetivo de avaliar se as variáveis e categorias de análise previamente estabelecidas foram adequadas para o problema de pesquisa ou se faltava algum critério. As categorias de análise finalmente utilizadas foram:

1. Empecilhos e facilitadores: a. empecilhos; b. facilitadores.

2. Uso de procedimento de ensino derivados da Análise do Comportamento: a. procedimentos utilizados; b. características dos procedimentos; c. tentativas de aplicação.

3. Situações subsequentes: a. eficácia de procedimentos; b. aceitação por parte de instituições de ensino.

4. Avaliação da situação da Análise do comportamento na educação e propostas de mudança: a. panorama da área; b. expectativas; c. propostas.

Para cada categoria de exame foi estabelecido um código. Em tabelas, os trechos que tratavam de alguma das categorias de exame foram copiados em uma coluna específica. Em tais trechos foi colocado um código com referência a que categoria cada trecho se enquadrava, podendo haver mais de uma. Em uma coluna intitulada "Observações do pesquisador" foram relatadas características das falas dos participantes com objetivo de facilitar a interpretação de cada trecho da entrevista categorizado e, assim, poder completar o exame das informações coletadas.

Os resultados foram enviados aos participantes e solicitado a eles que, num prazo de duas semanas, validassem as análises e exames, realizassem alterações ou pedissem um segundo momento de entrevista para complementar a entrevista passada. Para os casos em que houve total concordância com o que foi feito e não foram sugeridas alterações, o texto foi acrescentado à seção de resultados. Em casos em que os participantes sugeriram alterações, foram aceitas as mudanças e então acrescidas ao corpo dos resultados. A partir dos resultados de cada entrevista, os dados foram condensados em uma única análise.

\section{Resultados e Discussão}

\section{Empecilhos e Facilitadores}

Sobre os empecilhos para utilização dos procedimentos de ensino derivados da Análise do Comportamento, três participantes apontaram diretamente para o aumento da carga de trabalho do profissional como fator relevante. Um dos entrevistados afirmou ter tido dificuldades para aguentar o semestre inteiro com uma carga grande de trabalho, sem qualquer vantagem quer financeira quer de liberação de horas de trabalho, ganhando o mesmo que qualquer pessoa estava ganhando para fazer qualquer outra coisa. Ainda sobre o assunto, ao planejar o ensino com procedimentos analítico-comportamentais haveria maiores dificuldades com relação a feriados inesperados. Os procedimentos da área tornariam as disciplinas menos flexíveis.

Esses resultados estão de acordo com avaliação presente na literatura (Benjamin, 1988; Keller, 1983; Teixeira, 2006) em relação aos professores terem mais trabalho ao utilizarem procedimentos derivados dos conceitos da Análise do Comportamento do que seguindo métodos tradicionais de ensino. Vale ressaltar que esse maior custo de resposta pode ser uma variável importante para explicar a preferência, por parte dos educadores, por métodos de ensino que exigem menos esforços e dedicação.

O problema do aumento da carga de trabalho fica ainda mais acentuado pelo fato de as instituições de ensino proverem poucas condições para contratação de monitores. Quatro entre os cinco profissionais entrevistados indicaram esse aspecto. Dessa forma, a individualização do ensino, por exemplo, fica comprometida.

Há mais um empecilho relativo às condições dispostas ou impostas pelas instituições de ensino: o descompasso entre a quantidade de tempo necessária para que os alunos possam seguir em seus próprios ritmos e o tempo oferecido pelas universidades. Essa condição apareceu em todas as entrevistas realizadas, tendo como decorrência, o aumento da quantidade de trabalho e a necessidade de concessões: ensino é apenas possível em função das condições existentes, não chegando ao que seria necessário para uma efetiva aprendizagem. A falta 
de tempo oferecido pelas instituições de ensino aos professores também atrapalha o planejamento das disciplinas. Outra decorrência da escassez de tempo é a dificuldade para avaliação dos repertórios de entrada dos alunos.

Além da impossibilidade de contratação de monitores remunerados, outra condição também de natureza econômica, foi relatada. Nas experiências de dois entrevistados, os procedimentos analítico-comportamentais para ensino tiveram maiores custos financeiros. Um deles afirma que, para produzir bons materiais de ensino programado seria preciso contratar um programador de computador e fazê-los em versões computadorizadas, garantindo apresentação imediata das consequências e impossibilitando que os estudantes lessem as soluções corretas antes da apresentação das respostas. Isso teria um custo financeiro alto, de forma que fica pouco viável programar materiais nesse formato. Um outro participante ressaltou a necessidade de utilização de grande quantidade de materiais impressos para as atividades dos alunos, mas as instituições de ensino não fornecem a verba necessária para confecção dos mesmos.

De acordo com dois participantes, há necessidade da utilização, ao menos em parte, de materiais programados. Porém, é muito difícil desenvolver todos estes materiais para cada disciplina ministrada, de forma que são utilizadas partes de textos didáticos diferentes. Uma condição indicada como variável importante em dificultar a criação de textos programados é sua falta de valorização no âmbito acadêmico. Uma evidência disso é as instituições de fomento à pesquisa avaliarem pesquisadores - $\mathrm{e}$ fornecerem bolsas de apoio às pesquisas - apenas por artigos ou livros da área. Material didático não produz, pelo menos ao juízo das agências, o mesmo impacto na produção de conhecimento.

Skinner (1961) e Benjamin (1988) consideram que o aumento dos custos econômicos foi uma crítica recorrente ao uso da Instrução Programada e das máquinas de ensinar. Skinner argumentou que essa era uma crítica infundada, pois a longo prazo esses métodos tornariam possível o ensino de maior quantidade de alunos em menor tempo e por menos educadores. $\mathrm{O}$ mesmo argumento parece ser válido no caso de instituições em que as aulas tradicionais poderiam ser substituídas por tais métodos, o que parece ser uma realidade distante, já que há muita resistência ao uso desse tipo de procedimento.

Comportamentos baseados em concepções errôneas sobre Análise do Comportamento e Behaviorismo Radical apareceram em três entrevistas como barreiras para uso de procedimentos comportamentais de ensino. Em uma delas o entrevistado salienta que a concepção de explicações internalistas para a aprendizagem, por parte educadores, atrapalha na realização de intervenções em escolas, pois tais educadores creem que o aluno deve aprender sem ajuda externa ou programas computadorizados É importante salientar que, apesar dos preconceitos, não houve qualquer relato sobre não aceitação explícita do uso de procedimentos derivados da Análise do Comportamento nas instituições de ensino em que os participantes atuam como professores e orientadores. $\mathrm{O}$ que indica a existência de oportunidades para examinar a importância dessa condição na implementação de ensino com procedimentos e critérios comportamentais, visto que ela não foi citada por qualquer participante, embora apareça na literatura da área (Gioia, 2001; Miraldo, 1985; Rodrigues, 2005).

Surgem mais do que questões conceituais sobre a concepção de ensino nas escolas. No contexto escolar apareceram ainda relatos de impedimentos práticos para utilização de procedimentos computadorizados que facilitariam, de alguma forma, maior programação de condições de ensino coerentes com um "procedimento comportamental". Por exemplo, em escolas públicas, ao haver tentativas usar computadores, os professores se defrontam com grande incidência aparelhos defeituosos.

Sobre as dificuldades encontradas em derivar práticas educacionais a partir dos princípios da Análise do Comportamento, um dos entrevistados cita diversos empecilhos relativos à estrutura da universidade que resumem boa parte das dificuldades encontradas. Muita burocracia, resistência para alterar currículos e muitas demandas para os alunos dificultam as tentativas de uso de "procedimentos comportamentais" de ensino.

$\mathrm{O}$ fato de experientes analistas do comportamento encontrarem dificuldades em fazer uso de tecnologias da área leva a questionar as costumeiras afirmações de que a ciência do comportamento não encontra seu devido papel na educação por causa 
da ignorância ou preconceitos com as proposições da área. Ainda que elas encontrem respaldo em estudos da área (Rodrigues, 2005, 2012; Gioia, 2001), os prognósticos feitos são sombrio. O papel dos analistas do comportamento como vítimas pode ser questionado quando as maiores condições alegadas para não utilização dos procedimentos de ensino são relativos a características dos próprios procedimentos de ensino. Caberia aos analistas do comportamento o papel de questionar e planejar mudanças no curso dos estudos da área visando desenvolvimento de tecnologias mais simples e acessíveis para os professores utilizarem nas condições existentes nas instituições de ensino.

Grande carga de trabalho, procedimentos complicados e aumento dos custos econômicos tornam-se um problema crítico apenas porque há grande rigidez institucional. Caso as instituições de ensino oferecessem aos seus profissionais mais tempo e recursos pessoais e financeiros, melhorassem prazos e diminuíssem a burocracia, talvez os aspectos indicados como problemas característicos dos procedimentos de ensino não fossem problemas de fato. Assim, melhorar as condições também implica desenvolver procedimentos mais simples (que não esfacelassem a proposta, mas que fossem exequíveis no contexto existente) e trabalhar para melhores condições institucionais. Ambos os tipos de esforços poderiam produzir maior uso da tecnologia do ensino provida pela Análise do Comportamento.

\section{Uso de procedimentos de ensino derivados da Análise do Comportamento}

Quatro participantes informaram ter realizado programações de ensino nos moldes propostos por Carolina Bori em algum momento de suas carreiras. Devido às dificuldades encontradas, dois deles passaram a ser guiados pelos princípios básicos da área, mas com derivações informais dos princípios.

Para ambos participantes, o uso de procedimentos que considerassem o conceito de comportamento e fossem coerentes com os conceitos desenvolvidos com o conhecimento derivado da Análise do Comportamento se extinguiu tanto com o passar do tempo quanto com as barreiras impostas pela vida acadêmica. Essa extinção dos comportamentos profissionais necessários para manu- tenção do emprego dos conceitos produzidos pela área já foi salientada nos resultados encontrados por Maurutto (1999). O que parece indicar que as contingências vigentes não foram suficientes para a continuação do uso de procedimentos coerentes com o conhecimento da área e nem para o arranjo de novas contingências para que tais procedimentos se estabelecessem.

Dois outros participantes relataram seguirem utilizando procedimentos coerentes com o conhecimento da área nos seus cursos. Afirmaram utilizar ainda mais elementos de tecnologia de ensino, desenvolvidos a partir de pesquisas e experiências posteriores às de Carolina Bori, ainda que algumas concessões administravas precisassem ser feitas para se adequarem às exigências institucionais. Tais procedimentos de programação de condições para o desenvolvimento de comportamentos seria mais adaptável por poder ser utilizado por diferentes profissionais de ensino, contanto que alcançassem objetivos comportamentais propostos, o que também já seria parte de uma tecnologia comportamental e ainda é algo desconhecido e sequer cogitado pela quase totalidade de professores e administradores do ensino.

Para esses entrevistados tais procedimentos seriam utilizados primordialmente ou principalmente no período de programação das disciplinas, na fase anterior ao começo do semestre letivo dos estudantes. Já durante as aulas, haveria a necessidade de realizar algumas adaptações. Há ainda, para tais entrevistados, uma dificuldade para avaliar adequadamente os repertórios de entrada dos alunos. Além disso, por nem sempre conseguirem fazer todas as atividades de ensino programadas para todos os encontros, tendem a utilizar aulas expositivas em uma quantidade maior do que a desejável para ser coerente com um procedimento harmonioso com o conhecimento da área.

Em consonância com os princípios da Análise do Comportamento para a educação, tais entrevistados informaram solicitar tarefas semanais exigindo responder ativo dos alunos, além de avaliação e consequenciação das respostas apresentadas pelos aprendizes. Seriam feitos cursos "programados e individualizados o mais possível". Algumas concessões precisaram ser feitas, principalmente no que diz respeito ao ritmo de cada aluno, uma vez que há 
prazo determinado pela burocracia das instituições para a duração da disciplina.

É perceptível que para esses experientes analistas do comportamento as contribuições de Carolina Bori e os conceitos e procedimentos de ensino desenvolvido por ela (ou com ela) estão fortemente presentes em suas atuações docentes. O que confirma os argumentos apresentados por Nale (1998) sobre a importância de suas contribuições e sobre o fato de que a maior parte dos estudos e atuações em programação de condições para o desenvolvimento de comportamentos no Brasil tem influência de Carolina Bori.

Além desses critérios e procedimentos relacionados com a contribuição e influência de Carolina Bori, há relatos, por parte de três participantes, do uso de materiais de Instrução Programada. Por conta das dificuldades em escrever materiais deste tipo, os participantes disseram usar capítulos do livro escrito por Holland e Skinner (1961) sobre princípios básicos da Análise do Comportamento e um software para ensinar conceitos básicos.

Mesmo quando os conceitos e procedimentos desenvolvidos pela Análise do Comportamento não são diretamente utilizados, os participantes relatam derivarem seus procedimentos de ensino dos princípios da Análise do Comportamento. De maneira informal, com pouco rigor, os participantes dizem definir objetivos comportamentais para seus alunos e avaliá-los constantemente. Quatro entrevistados relataram seguir o princípio dos pequenos passos. Tal princípio estaria presente na escolha dos materiais didáticos e mesmo para guiar aulas expositivas. Além disso, todos os participantes afirmaram provocar ou solicitar respostas abertas, seguindo a ideia do responder ativo.

Para um dos participantes, todos os procedimentos derivados dos conceitos da área, mesmo que não propostos diretamente para uso educacional, podem fazer parte de qualquer procedimento de programação de condições para o desenvolvimento de comportamentos em contexto de ensino. De acordo com essa concepção, o entrevistado relata usar diversas técnicas da área em seus procedimentos como professor: fading, treino discriminativo, modelagem, modelação, matching to sample etc. Ele vai além e afirma que quaisquer procedimentos podem ser condizentes com os princípios da área, contanto que conceitos básicos sobre o comportamento humano estejam sendo respeitados.

Mesmo quando há impossibilidades para aplicação dos procedimentos de ensino da área, os profissionais seguem atuando de forma condizente com a teoria. De fato, três participantes afirmam não conseguir mais utilizar os procedimentos de forma integral, enquanto dois afirmam usar com limitações, o que comprova a existência das dificuldades já indicadas na utilização da tecnologia comportamental para ensino. O que leva a considerar que ainda precisa ser examinado e avaliado quanto o ensino baseado nos princípios e procedimentos da área, mesmo que não sejam utilizados procedimentos integralmente coerentes com princípios derivados do conhecimento a respeito dos processos relacionados ao desenvolvimento de comportamento operante no contexto formal, são suficientes para promoção do ensino eficaz.

Tais procedimentos de ensino, quando baseados em princípios da Análise Experimental do Comportamento e coerentes com tais princípios parecem ser uma alternativa viável para manter as assunções básicas da área sendo conhecidas e demonstrando sua contribuição e validade em contextos de ensino mais comuns ou usuais É possível que tais procedimentos produzam bons resultados, porém, precisam ser acompanhados de demonstrações inequívocas para que possam ser considerados como pertinentes. É a própria coerência instrumental com os conceitos e princípios básicos derivados da Análise Experimental do Comportamento e a avaliação do que resulta de um trabalho ou procedimento com tal coerência que constitui a base para afirmar que qualquer procedimento seja "comportamental”. Só com essa condição qualquer experiência poderia fornecer dados para indicar se há eficácia em tais "experiências". Em caso afirmativo tais avaliações e demonstrações inequívocas poderiam servir como ferramenta a outros profissionais da educação comprometidos com a concepção behaviorista radical em relação ao ensino formal.

\section{Situações Subsequentes}

Ao tratarem dos resultados encontrados, todos os participantes afirmaram ter eficácia em seus procedimentos de ensino. Isso ocorreu tanto para os entrevistados em que as situações nas quais os pro- 
cedimentos de ensino já configurados como da área foram usados quanto para quando os princípios foram utilizados com procedimentos não usualmente conhecidos como "da área". O abandono do uso de procedimentos "consagrados" na área não foi, em entrevista alguma, indicado como efeito de maus resultados, mas sim por motivos outros, o que pode incluir também aperfeiçoamento dos procedimentos usuais da área ou criação de novos procedimentos e tecnologias.

Ao utilizar tecnologias de ensino Maurutto (1999) constatou que a aprovação social da comunidade de analistas do comportamento e o próprio progresso de seus alunos eram considerados consequências reforçadoras para os participantes de sua pesquisa. É preciso, de acordo com essa constatação, questionar ou verificar com grande cuidado as contingências que fazem com que "valha a pena" seguir alguns princípios da área, mas manter diversos elementos do ensino tradicional como pode ser o caso de adaptações e improvisos superficialmente denominados de "comportamentais". Em outras palavras, é necessário comparar a qualidade dos resultados no desenvolvimento de comportamentos (um suposto reforçador) do aluno quando são e quando não são usados procedimentos considerados "da área”. Também parece ser importante avaliar por quais motivos a aprovação social da comunidade não seleciona e mantém comportamentos de aplicar tecnologias de ensino efetivamente comportamentais. Se houvesse maior densidade de reforçamento social para comportamentos de usar procedimentos de ensino coerentes com o conhecimento, conceitos e princípios da área do que para respostas com diferentes topografias, então poderia haver maiores chances de os membros da comunidade de analistas do comportamento preferirem procedimentos mais condizentes com as contribuição de conhecimento da Análise do Comportamento. O que sempre precisará ser feito em conjunto com os exames e avaliações envolvendo os custos de respostas e os empecilhos existentes para comportamentos profissionais coerentes com o conhecimento da área.

Foram apresentadas nas entrevistas algumas avaliações negativas por parte dos estudantes ao serem submetidos a procedimentos de ensino considerados como sendo de "abordagem comportamental". Após uma das experiências com um pro- cedimento apresentado como ensino programado, houve a preferência dos alunos pelos métodos tradicionais de ensino, por considerarem que havia maior controle e rigidez no que foi denominado por "ensino programado". Outro participante relatou que entre as críticas negativas recebidas dos alunos estão as de que tal procedimento obriga a muito trabalho, muita exigência e pouco tempo para estudar, devido à quantidade de tarefas exigidas. O que também produz grande quantidade de desistências.

Os dados observados são convergentes tanto com aqueles encontrados por Miraldo (1985), quanto por Miller e Mallot (2006). Miraldo (1985) recolheu avaliações em que os alunos achavam os cursos da área metódicos, exaustivos e rígidos. Miller e Mallot (2006) ouviram relatos contrários dos alunos quanto ao procedimento de Instrução Programada (IP) durante estudo sobre tal procedimento e indicaram a importância de obter opiniões dos alunos; consideraram que para aumentar as chances de sucesso das tecnologias de ensino da área, é importante a aprovação do público alvo.

Por outro lado, houve relatos por parte de dois participantes de que os alunos passaram a se interessar mais por Análise do Comportamento após serem alunos de disciplinas da área. Ao mesmo tempo em que há críticas à demasia de tarefas e rigidez produzidas por procedimentos 4analítico-comportamentais de ensino, há elogios à seriedade e efetividade dos mesmos.

\section{Avaliação da Situação da Análise do Comportamento na Educação e Propostas de Mudança}

Como já dito, o alto custo de resposta para preparação de material de ensino quando há procedimentos de programação de condições de ensino coerentes com o conhecimento de Análise do Comportamento é um empecilho para o uso e difusão de tais procedimentos entre professores, até da própria área de conhecimento. Por considerar importante a produção de materiais programados na realização do trabalho de programação de desenvolvimento de comportamentos, um dos participantes sugere que os analistas do comportamento se organizem para preparação de materiais de instrução programada: alguém (ou um grupo) pode 
trabalhar com um aspecto (por exemplo, um dos conceitos de análise do comportamento), outros com diferentes aspectos. Assim, haveria materiais programados para o ensino dos principais conceitos da área ou contribuições diversas (técnicas, procedimentos, princípios etc.).

Outra sugestão contida nas entrevistas diz respeito à possibilidade de contornar a resistência de profissionais da educação. Se os analistas do comportamento fornecessem procedimentos simples e claramente eficazes que, além de não aumentarem a carga de trabalho, funcionem, com efetivos resultados relevantes então as resistências seriam diminuídas. Caberia aos analistas do comportamento o papel de desenvolver procedimentos simples e de fácil realização e demonstrar claramente as vantagens deles.

Para um dos participantes, os analistas do comportamento têm trabalhado de forma eficaz com educação. Ele considera que há uma geração que teve contato com cursos sobre programação de ensino, principalmente com Carolina Bori, que mantém parte daquilo estudado sobre programação de ensino em seu trabalho como educadores. Há casos de analistas do comportamento que desenvolveram tecnologias no que pode ser considerado "programação de ensino". Por outro lado, aponta que novas gerações de analistas do comportamento têm perdido o contato com as propostas de programação de ensino. Apesar disso, acredita que o conhecimento de conceitos básicos e procedimentos da área já os tornariam mais aptos para realização de um ensino efetivo.

Outra opinião apresentada nas entrevistas é a de que há pouca correspondência entre o discurso e os procedimentos de grande parte dos analistas do comportamento. Um entrevistado considera que há insuficiente correspondência entre o discurso e os procedimentos de ensino mesmo entre analistas do comportamento. Ainda há distância entre os conceitos disponíveis e os procedimentos utilizados: por exemplo, aulas ainda são seriam dadas de maneira tradicional. Parece haver ainda poucos analistas do comportamento planejando disciplinas baseadas em programação de condições para desenvolvimento de comportamentos relevantes. Usualmente as aulas seriam preparadas com base em textos a respeito de Análise do Comportamento para ler e discutir com os alunos.
Além disso, é considerado haver falta de um projeto conjunto entre os programas de pós-graduação que formam analistas do comportamento para definição de comportamentos necessários para os alunos. Tal consideração é estendida aos congressos da Associação Brasileira de Medicina e Psicologia Comportamental (ABPMC), nos quais os cursos oferecidos não seriam programados para atualizar e formar pessoas de modo progressivo, cumulativo ou articulado; o que ocorre é que cada um propõe e ministra o curso a respeito do que sabe, ou considera importante ou está realizando como trabalho de investigação, mais de forma expositiva do que programada para desenvolver comportamentos de interesse. Por fim, salienta um entrevistado, parece ser necessário os analistas do comportamento agirem de forma mais condizente com os princípios básicos da Análise do Comportamento para mudar esse contexto predominante no meio educacional. Inclusive nas intervenções feitas, que parecem ser pouco experimentais e sem demonstrações apropriadas dos efeitos ou resultados dos procedimentos de intervenção.

Há autores que indicam possibilidades para alterar as condições a que os entrevistados se referem e, assim, facilitar a disseminação das tecnologias de ensino nos ambientes e instituições educativas. Deitz (1994) sugere algumas providências a serem tomadas em relação à própria comunidade de analistas do comportamento e à sociedade como um todo: (a) coletar mais dados com maior quantidade de alunos, disciplinas e idades; (b) investigar temas relevantes para o ensino regular: diversidade cultural, autoestima, avaliação de desempenho etc.; (c) participar diretamente em treinamento de professores; (d) estudar em instituições de ensino voltadas para a educação como forma de aproximar os analistas do comportamento dos educadores; (e) apresentar dados para audiências de não analistas do comportamento; (f) utilizar os conhecimentos sobre análises de contingências para agir melhor nessas situações; (g) formar parcerias com instituições de ensino preocupadas em métodos baseados em evidências; (h) trabalhar politicamente, com engajamento em organizações envolvidas em assuntos educacionais. Outras sugestões foram dadas por Fantuzzo e Atkins (1992) quando sugerem 
que estudos da área devem produzir métodos que considerem a diversidade de características dos estudantes encontrados por professores no ensino regular e as restrições institucionais e burocráticas existentes.

As recomendações dos autores convergem com algumas sugestões dos participantes da pesquisa na medida em que apontam para procedimentos que se adequem à estrutura vigente, ao diálogo com outros educadores e ao engajamento político.

Em síntese, considerando que o objetivo do trabalho foi investigar questões envolvidas na aplicação de procedimentos de ensino da Análise do Comportamento, o acesso ao relato verbal por meio de entrevistas de analistas do comportamento foi considerado uma estratégia adequada e, provavelmente, a melhor possível para atender ao problema de pesquisa. $\mathrm{O}$ procedimento de fornecer as análises prévias aos participantes mostrou-se importante no sentido de refinar as informações obtidas durante as entrevistas.

Os empecilhos para uso dos procedimentos de ensino encontrados durante as entrevistas merecem posterior discussão e aprofundamento. Foram elencadas diversas barreiras, porém há dificuldades para estabelecer o quanto e de que forma cada uma delas influencia na pouca utilização de procedimentos de ensino coerentes com o conhecimento produzido na área. Um trabalho que preencha tal lacuna de conhecimento e tecnologia provavelmente será de grande auxílio para definir prioridades na solução dos problemas identificados pelos entrevistados.

No presente trabalho, foram obtidos depoimentos a respeito de empecilhos e de resultados do "uso de procedimentos de ensino derivados da Análise do Comportamento". Em diversos momentos, as informações obtidas por meio dos entrevistados foram pouco diferenciadas, no sentido em que tratam do uso de quaisquer métodos, técnicas e procedimentos considerados como derivados dos conhecimentos da área. Assim, há um grande perigo ao falar das dificuldades em utilizar procedimentos educacionais da Análise do Comportamento de forma geral, desconsiderando diferenças entre variados procedimentos de ensino utilizados por analistas de comportamento ou mesmo por professores não analistas do comportamento.
Parece terem surgido contribuições para o trabalho com Programação de Ensino, pois os dados dizem respeito a procedimentos utilizados por analistas do comportamento na educação e indicam falhas e alternativas. Fica em aberto, porém, uma ainda grande necessidade de que novos estudos examinem e discutam os trabalhos de programação de ensino, salientando as dificuldades relativas às possíveis experiências com esse tipo de trabalho. Considerando a importância dos conceitos e procedimentos de ensino desenvolvidos por Carolina Bori no Brasil (Nale, 1998) e o fato de a maior parte dos entrevistados terem sido influenciados por ela, indica a importância de estudos desse tipo lidando com as dificuldades para implementação de cursos feitos a partir da análise de contingências em programação de condições para o desenvolvimento de comportamentos.

Ainda tratando sobre a programação de condições para o desenvolvimento de comportamentos, pelo menos o que foi desenvolvido no Brasil, parece haver necessidade de desenvolvê-la e divulgá-la de forma mais ostensiva e clara. Afinal, alguns participantes levantaram o problema de que as gerações que tiveram contato direto com Carolina Bori carregam em seus trabalhos profissionais as influências de suas contribuições relativas aos processos de ensino e aprendizagem, mas as novas gerações de analistas do comportamento estão se distanciando dessas contribuições talvez por mero desconhecimento. Parece haver um distanciamento entre contribuições e conhecimento de diferentes gerações. Tal distanciamento parece exigir, no mínimo, atividades como cursos, palestras, artigos, livros etc. a respeito da história de desenvolvimento e das contribuições do que pode ficar sob a denominação de "programação de condições de ensino".

É importante salientar que o fato de diferentes procedimentos de ensino já formulados e descritos não estarem sendo devidamente utilizados não necessariamente os invalida ou os torna pouco importantes. Sua utilização mostra que eles têm eficácia considerável e, por isso, precisam ser valorizados e difundidos. Os relatos de trabalhos que dão certo demonstram que podem ser ferramentas úteis para lidar com os processos de ensino aprendizagem. Porém, como já foi examinado por Baer, Wolf e Risley $(1968 ; 1987)$, é importante levar em conta 
as possibilidades de utilização nos contextos concretos em que precisam ou podem ser utilizados.

Há diversas possibilidades de contribuição dos analistas do comportamento que atuam e pesquisam com relação às relações entre os processos ensinar e aprender no sentido de promoverem o desenvolvimento de procedimentos demonstradamente eficazes fundamentados em princípios da Análise do Comportamento. Possíveis tipos de trabalho são: desenvolver procedimentos e técnicas facilmente aplicáveis em salas de aula com custo de resposta mais baixo para os educadores; analisar as contingências em que os analistas do comportamento estão inseridos em âmbitos social, político e institucional e, como decorrência, planejar mudanças em ambientes educacionais; difundir procedimentos educacionais efetivos que sejam simples, com linguagem acessível em diálogo com educadores. Enfim, parece ser útil examinar a necessidade de aperfeiçoamentos nas atuações dos analistas do comportamento em educação, "Ou, poderemos continuar produzindo [apenas] dentro da universidade, para que a universidade nos avalie e mantenha (ou não) o nosso status" (Luna, 2001, p. 155).

\section{Referências}

Araújo, S. L. (2008). Educação a distância com um sistema personalizado de ensino (Dissertação de Mestrado). Pontifícia Universidade Católica de São Paulo, São Paulo, SP.

Axelrod, S. (1992). Disseminating an effective educational technology. Journal of Applied Behavior Analysis, 25(1), 31-35.

Baer, D. M., Wolf, M. M., \& Risley, T. R. (1968). Some current dimensions of applied behavior analysis. Journal of Applied Behavior Analysis, $1(1), 91-97$.

Baer, D. M., Wolf, M. M., \& Risley, T. R. (1987). Some still current dimensions of applied behavior analysis. Journal of Applied Behavior Analysis, 20(4), 313-327.

Benjamin, L. T. (1988). A history of teaching machines. American psychologist, 43(9), 703-712.

Bijou, S. W. (1970). What psychology has to offer education- now. Journal of Applied Behavior Analysis, 3(1), 65-71.
Cortegoso, A. L., \& Coser, D. S. (2011). Elaboração de programas de ensino: Material autoinstrutivo. São Carlos, SP: Edufscar.

Crosbie, J., \& Kelly, G. (1994). Effects of imposed postfeedback delays in programmed instruction. Journal of Applied Behavior Analysis, 27(3), 483-491.

Deitz, S. M. (1994). The insignificant impact of behavior analysis on education: notes from a dean of education. Em R. Gardner, D. Sainato, J., Cooper, T. E. Heron, W. L. Heward, J. W. Eshleman, \& T. A. Grossi (Eds.), Behavior analysis in education: Focus on measurably superior instruction (pp. 33-41). Pacific Grove, CA: Brooks/Cole.

Fantuzzo, J., \& Atkins, M. (1992). Applied behavior analysis for educators: Teacher centered and classroom based. Journal of Applied Behavior Analysis, 25(1), 37- 42.

Fredrick, L. D., \& Hummel, J. H. (2004). Reviewing the outcomes and principles of effective instruction. Em D. J. Moran \& R. W. Malott (Eds.), Evidence-based educational methods: Advances from the behavioral sciences (pp. 9-22). San Diego, CA: Elsevier Academic Press.

Gioia, P., S. (2001) A abordagem behaviorista radical transmitida pelo livro de psicologia direcionado à formação de professores (Tese de Doutorado) Pontifícia Universidade Católica de São Paulo, São Paulo, SP.

Heward, W. L. (2005). Reasons applied behavior analysis is good for education and why those reasons have been insufficient. Em W. L. Heward, T. E. Heron, N. A. Neef, S. M. Peterson, D.M. Sainato, G. Cartledge, R. Gardner III, L. D. Peterson, S. B. Hersh, \& J. C. Dardig (Eds.), Focus on behavior analysis in education: Achievements, challenges, and opportunities (pp. 316-348). Upper Saddle River, NJ: Merrill/ Prentice Hall.

Holland, J. G. (1960). Teaching machines: an application of principles from the laboratory. Journal of the Experimental Analysis of Behavior, 3(4), 275-287.

Holland, J. G. (1967). A quantitative measure for programmed instruction. American Educational Research Journal, 87-101. 
Holland, J. G. (1978). Behaviorism: part of the problem or part of the solution. Journal of Applied Behavior Analysis, 11(1), 163-174.

Holland, J. G. \& Skinner, B. F. (1961). The analysis of behavior. New York: McGraw- Hill Book Company.

Juliano, M. C.; Luna, S. V. (2008) Programação de ensino como uma tecnologia para a educação. Em SILVA, W. C. M. P. (Eds.), Sobre comportamento e cognição (Vol. 21, pp. 219- 233). Santo André, SP: Esetec.

Keller, F. S. (1968). “Good bye, teacher...”. Journal of Applied Behavior Analysis, 1(1), 79-89.

Keller, F. S. (1983). Aprendendo a ensinar: memórias de um professor universitário. São Paulo, SP: Edicon.

Keller, F. S. \& Sherman, J. G (1974). PSI, the Keller Plan Handbook: Essays on a personalized system of instruction. Menlo Park, California: WA Benjamin.

Kienen, N., Kubo, O. M., \& Botomé, S. P. (2013). Ensino programado e programação de condições para o desenvolvimento de comportamentos: alguns aspectos no desenvolvimento de um campo de atuação do psicólogo. Acta Comportamentalia, 21(4), 481-494.

Kubo, O. M., \& Botomé, S. P. (2001). Ensinoaprendizagem: uma interação entre dois processos comportamentais. Interação em Psicologia, $5,133-171$

Lacerda, D. F. de (2008). Caracterização do ensino programado no Brasil: um estudo com base na análise de periódicos científicos (Dissertação de Mestrado). Pontifícia Universidade Católica de São Paulo, São Paulo, SP.

Luna, S. V. (1998) Planejamento de pesquisa: elementos para uma análise metodológica. São Paulo, SP: EDUC.

Luna, S. V. (2001). A crise da educação e o Behaviorismo. Que parte nos cabe nela? Temos soluções a oferecer? Em: K. Carrara (Org.), Educação, Universidade e Pesquisa. III Simpósio em Filosofia e Ciência: Paradigmas do Conhecimento no Final do Milênio (p. 143155). São Paulo, SP: FAPESP/ Unesp-MaríliaPublicações.

Maurutto, A. A. (1999). Revisitando a programação do ensino no Brasil (Dissertação de Mestrado).
Pontifícia Universidade Católica de São Paulo, São Paulo, SP.

Miller, M. L., \& Malott, R. W. (2006). Programmed instruction: Construction responding, discrimination responding, and highlighted keywords. Journal of Behavioral Education, 15(2), 109117.

Miraldo, C. M. V. (1985). Conhecimento e crenças de estudantes de Psicologia acerca da Análise Experimental do Comportamento. (Dissertação de Mestrado) Universidade de São Paulo, São Paulo, SP.

Moran, D. J. (2004). The need for evidence-based educational methods. Em D. J. Moran \& R. W. Malott (Eds.), Evidence-based educational methods: Advances from the behavioral sciences (pp. 3-7). San Diego, CA: Elsevier Academic Press.

Moreira, M. B. (2004). Em casa de ferreiro, espeto de pau: o ensino de análise experimental do comportamento. Revista Brasileira de Terapia Comportamental e Cognitiva, 6(1), 73-79.

Nale, N. (1998). Programação de ensino no Brasil: O papel de Carolina Bori. Psicologia Usp, 9(1), 275-301.

Rodrigues, M. E. (2005). A contribuição do Behaviorismo Radical para a formação de professores-uma análise a partir das dissertações e teses no período de 1970 a 2002 (Tese de Doutorado). Pontifícia Universidade Católica de São Paulo, São Paulo, SP.

Rodrigues, M. E. (2012). Behaviorismo Radical, análise do comportamento e educação: o que precisa ser conhecido? Em: Carmo, J. S. \& Ribeiro, M. J. (Orgs.) Contribuições da análise do comportamento à prática educacional. Santo André, SP: ESETec.

Rumph, R., Ninness, C., McCuller, G., Holland, J., Ward, T., \& Willbourn, T. (2007). “The Shame of American Education" Redux. Behavior and Social Issues, 16(1), 27-41.

Santos, B. C. dos (2012). O estudo do controle aversivo no Brasil com base em teses e dissertações: uma perspectiva histórica (Dissertação de Mestrado). Pontifícia Universidade Católica de São Paulo, São Paulo, SP.

Skinner, B. F. (1958). Teaching machines. Science, 128, 969-977. 
Skinner, B. F. (1961). Why we need teaching machines. Harvard Educational Review, 31, 377-398.

Skinner, B. (1963). Reflections on a decade of teaching machines. The Teachers College Record, 65(2), 168-177.

Skinner, B. F. (1968). Teaching Science in High School-What is Wrong? Science, 159, 704-710.

Skinner B. F. (1972) Some implications of making education more efficient. In: C. E.

Thoresen (ed.) Behavior modification in education. Chicago: National Society for the Study of Education, 446- 456.

Skinner, B. F. (1974a). The free and happy student. Readings in Curriculum and

Supervision, 55(1), 97.

Skinner, B. F. (1974b). About Behaviorism. New York: Alfred A. Knopf.

Skinner, B. F. (1984). The shame of American education. American Psychologist, 39(9), 947-54.

Skinner, B. F. (1991). Questões recentes na análise comportamental (Tradução de A. L. Néri) Campinas, SP: Papirus Editora. (Trabalho original publicado em 1989)

Skinner, B. F. (2003). Ciência e comportamento humano. São Paulo: Martins Fontes.

(Trabalho original publicado em 1953).

Stromer, R., Mackay, H. A., \& Stoddard, L. T. (1992). Classroom applications of stimulus equivalence technology. Journal of Behavioral Education, 2(3), 225-256.

Teixeira, A. M. S. (2006). Análise de contingências em Programação de Ensino

Infantil-liberdade e efetividade na Educação. Santo André, SP: ESETec.

Tudor, R. M. (1995). Isolating the effects of active responding in computer-based instruction. Journal of Applied Behavior Analysis, 28(3), 343-344.

Vargas, E. A., \& Vargas, J. S. (1991). Programmed instruction: What it is and how to do it. Journal of Behavioral Education, 1(2), 235-251.
Informações do Artigo

Histórico do artigo:

Submetido em: 19/05/2015

Primeira decisão editorial: 24/06/2015

Aceito em: 04/08/2015

Editor Associado: Nicodemos Borges 